\title{
Effects of Sex and Weather on the Risk of Incidence of Anti-EGFR Therapy-Induced Rash in Cancer Patients: A Retrospective Study.
}

\section{Takahiro Arai}

Division of Pharmacy, Gunma Prefectural Cancer Center, Ota, Gunma, Japan

\section{Yukiyoshi Fujita ( $\nabla$ yfujita@gunma-cc.jp)}

Division of Pharmacy, Gunma Prefectural Cancer Center, Ota, Gunma, Japan

\section{Hisao Imai}

Department of Respiratory Medicine, Comprehensive Cancer Center, International Medical Center,

Saitama Medical University, Hidaka, Saitama, Japan

\section{Hiroe Matsumoto}

Division of Nursing, Gunma Prefectural Cancer Center, Ota, Gunma, Japan

\section{Miho Yamazaki}

Division of Nursing, Gunma Prefectural Cancer Center, Ota, Gunma, Japan

\section{Eriko Hiruta}

Division of Pharmacy, Gunma Prefectural Cancer Center, Ota, Gunma, Japan

\section{Yuka Suzuki}

Division of Pharmacy, Gunma Prefectural Cancer Center, Ota, Gunma, Japan

\section{Hitoshi Ojima}

Department of Gastroenterological Surgery, Gunma Prefectural Cancer Center, Ota, Gunma, Japan Hisashi Hosaka

Division of Gastroenterological Medicine, Gunma Prefectural Cancer Center, Ota, Gunma, Japan

\section{Koichi Minato}

Division of Respiratory Medicine, Gunma Prefectural Cancer Center, Ota, Gunma, Japan

\section{Taeko Saito}

Division of Pharmacy, Gunma Prefectural Cancer Center, Ota, Gunma, Japan

\section{Research Article}

Keywords: cancer, Seasonal climatic changes, skin rashes, effects of ultraviolet light, lack of skincare, male hormones

Posted Date: May 17th, 2021

DOI: https://doi.org/10.21203/rs.3.rs-523235/v1 
License: (c) (i) This work is licensed under a Creative Commons Attribution 4.0 International License. Read Full License 


\section{Abstract}

Seasonal climatic changes may affect the development of the rash that is characteristic of treatment with anti-epidermal growth factor receptor (EGFR) antibodies. This study evaluated the association between seasons and rash incidence among patients with cancer in Japan. Data of patients with colorectal or head and neck cancer treated with cetuximab or panitumumab during summer (S group; $\mathrm{N}=$ 34) or winter ( $W$ group; $N=37$ ) between June 2014 and February 2019 were collected to retrospectively examine patient characteristics and rash incidence for $\leq 8$ weeks after treatment initiation. Rashes were observed in $73.5 \%(\mathrm{~N}=25)$ and $78.4 \%(\mathrm{~N}=29)$ and grade 3 rashes were observed in $17.6 \%(\mathrm{~N}=6)$ and $2.7 \%(\mathrm{~N}=1)$ of the patients in the $\mathrm{S}$ and $\mathrm{W}$ groups, respectively, indicating higher rash incidence during summer $(p=0.09)$. Incidence of grade $\geq 2$ rash in men in the $S$ group was higher than that in the rest of the patient groups $(p<0.01)$, suggesting that rashes were more severe in men during summer. The higher incidence of skin rashes in men during summer might be attributed to the effects of ultraviolet light, lack of skincare, male hormones, and secretion of anti-EGFR antibodies in sweat. These findings highlighted the need for research on preventive measures for such rashes.

\section{Introduction}

Skin toxicity is one of the common adverse effects of cancer chemotherapy and is manifested in several ways, such as erythema over palmoplantar surfaces, rash, dry skin, and pruritus. Although skin toxicity is not life-threatening, it affects the daily life of severe cases and leads to dose reduction or cancer chemotherapy discontinuation [1].

Skin rash is frequently associated with the administration of epidermal growth factor receptor (EGFR) inhibitors, including tyrosine kinase inhibitors, such as gefitinib and erlotinib, and anti-EGFR monoclonal antibodies, such as cetuximab and panitumumab [2]. EGFR tyrosine kinase inhibitors are mainly used to treat lung cancer. In contrast, anti-EGFR monoclonal antibodies are essential drugs used for colorectal cancer (CRC) and head and neck cancer (HNC). Prophylactic treatment using skin moisturizers, sunscreens, topical steroids, and tetracycline antibiotics can reduce the incidence of skin toxicity due to exposure to anti-EGFR monoclonal antibodies $[3,4]$. Rash severity has been positively correlated with the clinical outcomes of EGFR inhibitor administration in several cancer types $[5,6]$. Therefore, it is critical to optimize the prophylactic management of skin toxicity induced by such treatments.

As defined by the Japan Meteorological Agency, the four seasons in Japan are Spring (March to May), Summer (June to August), Autumn (September to November), and Winter (December to February) [7]. Various climatic changes characterize the four seasons. For instance, in Maebashi City, Gunma Prefecture, the average temperature and humidity from 2014 to 2019 were $25.2{ }^{\circ} \mathrm{C}$ and $72.2 \%$ in summer and $5.0^{\circ} \mathrm{C}$ and $53.7 \%$ in winter, respectively. The changes in temperature and humidity for each season according to the Japan Meteorological Agency are shown in Fig. 1a [8]. Owing to these climatic changes, in Japan, the water content of the skin's stratum corneum in healthy subjects has been reported to decrease significantly in winter compared to that in summer [9]. Furthermore, atopic dermatitis symptoms 
are worse during winter [10]. These seasonal changes are considered to be a result of low humidity and low-temperature environments and decreased skin barrier function [11]. Therefore, it may be hypothesized that skin toxicity due to cancer chemotherapy may vary on the basis of seasons and sex. For instance, a previous report that studied the relationship between skin toxicities and seasons showed that the incidence of a hand-foot syndrome caused by sunitinib was higher in summer than in other seasons [12]. Furthermore, the EVITA trial, which examined the effect of a vitamin K1-containing cream on cetuximabinduced rash, showed a preventive effect only in women [13]. The findings of this trial indicated that men may have a higher incidence of rash than women, and there may be sex-related differences in the occurrence of rash. However, the relationship between EGFR inhibitor-induced rash and seasons or sex has not been investigated.

This study aimed to analyze the relationship between rash incidence and seasonal changes and sex in patients with CRC or HNC treated with anti-EGFR monoclonal antibodies.

\section{Methods}

\section{Ethical approval}

The Gunma Prefectural Cancer Center Ethics Committee approved this study on February 26, 2020 (Ethics code: 405-31079) and waived the requirement for informed consent owing to the retrospective nature of the study. All procedures performed in this study were in accordance with the ethical standards of the institutional research committee and the Declaration of Helsinki (1964) and its later amendments or comparable ethical standards.

\section{Season definition and patients}

This study retrospectively reviewed the cohort of consecutive patients who received cetuximab or panitumumab for CRC or HNC, during summer or winter, between June 1, 2014, and February 28, 2019. Cetuximab $\left(500 \mathrm{mg} / \mathrm{m}^{2}\right)$ or panitumumab $(6 \mathrm{mg} / \mathrm{kg})$ were administered every 2 weeks in patients with CRC. Cetuximab was administered at an initial loading dose of $400 \mathrm{mg} / \mathrm{m}^{2}$ and a maintenance dose of $250 \mathrm{mg} / \mathrm{m}^{2}$ once a week in patients with HNC. On receiving treatments, patients were instructed by pharmacists on the need to moisturize, protect, and cleanse the skin. And when the treatment was continued, the nurse checked the patient's skin condition and explained the importance of skin care to the patients. Patients who received treatment during summer were assigned to the $\mathrm{S}$ group, and those who were treated in winter were assigned to the $\mathrm{W}$ group. Summer was defined as the period from June to August, and winter was the period from December to February. Patients who received EGFR inhibitors within 1 year prior after the start of treatment with cetuximab or panitumumab, who discontinued treatment with anti-EGFR antibodies within two courses, and/or who received regular oral steroids prior to treatment were excluded from the study. 


\section{Evaluation method}

We retrospectively examined the sex, age, body surface area, treatment dose, Eastern Cooperative Oncology Group performance status, and rash incidence of all patients using data extracted from their medical information records. The primary endpoint was the incidence of a skin rash. The incidence and severity of rash were evaluated up to 8 weeks after the start of treatment with anti-EGFR antibodies. The severity of the rash during every treatment cycle was evaluated based on the Common Terminology Criteria for Adverse Events, version 4.0. The time until incidence and severity of the rash were determined using patient medical records and medical staff evaluations.

\section{Statistical analyses}

Continuous variables were presented as medians (ranges) and categorical variables as percentages (\%). The continuous variables were compared using the Mann-Whitney $U$ test, and those between multiple groups were compared using the Kruskal-Wallis test. Categorical variables were compared using the $\chi^{2}$ test. When expected cell counts were found to be less than five in the contingency table, Fisher's exact test with Yate's continuity correction was used. Time-to-event endpoints were analyzed using the KaplanMeier method and compared using the log-rank test. Hazard ratios were calculated using a Cox proportional hazard model. All analyses were performed using StatMate V (Atoms, Tokyo). A two-tailed $p$ value $<0.05$ was considered indicative of statistical significance. Based on previous studies, we assumed that the incidence of all grades of rash would be $70 \%$ in summer and $35 \%$ in winter $[4,8,10]$. Sixty-two patients were required to detect a difference in rash incidence between the groups with a power of $80 \%$ and a significance value of 0.05 . Assuming that some data would be missing in $10 \%$ of the patients, a sample size of 69 was determined.

\section{Results}

\section{Patient characteristics}

We identified 71 patients treated with cetuximab or panitumumab for CRC or HNC during the summer (N $=34)$ and winter $(\mathrm{N}=37)$ seasons and these patients were assigned to the $S$ and $W$ groups, respectively. None of these patients met the exclusion criteria (Fig. 2). The characteristics of the patients in both groups were comparable (Table 1).

\section{Seasonal differences in skin toxicities}

All grades of rashes were observed in 73.5\% $(\mathrm{N}=25)$ and $78.4 \%(\mathrm{~N}=29)$ of the patients in the $\mathrm{S}$ and $\mathrm{W}$ groups, respectively; however, no significant difference was observed between the two groups $(p=0.63)$ (Fig. 3a). Grade 3 rash was present in $17.6 \%(\mathrm{~N}=6)$ and $2.7 \%(\mathrm{~N}=1)$ of the patients in the $S$ and $\mathrm{W}$ 
groups, respectively, indicating increased frequency during summer; however, the intergroup difference was not significant $(p=0.09)$ (Fig. 3a).

\section{Sex-related differences in skin toxicities}

All grades and grade $\geq 2$ rash were observed in $81.3 \%(\mathrm{~N}=38)$ and $52.2 \%(\mathrm{~N}=24)$ of men, and $64.0 \%(\mathrm{~N}$ $=16)$ and $20.0 \%(\mathrm{~N}=6)$ of women, respectively, with a significant difference in rash severity between the two groups $(p=0.02)$ (Fig. 3b). In addition, we analyzed the association between sex and frequency of rashes in both groups. Our analysis showed that the frequency of grade $\geq 2$ rash was significantly higher in men in group $S$ than in any other group $(p<0.01)$ (Fig. 4). However, there was no significant difference with respect to the rash incidence frequency between men and women in group W (Fig. 4).

\section{Discussion}

Our results did not indicate any significant differences in the incidence of rashes upon treatment with anti-EGFR antibodies between the $S$ and $W$ groups. On the other hand, although a sex-based evaluation suggested that men were significantly more prone to skin rash than women in summer, there was no significant association between the incidence of rash and sex of the patients treated in winter. The tissue concentrations of EGF and EGF receptors are regulated by sex hormones such as estrogen and testosterone, which may contribute to sex differences in the development of skin rashes induced by antiEGFR antibodies [14]. The higher incidence of skin rashes in men during summer may be attributed to the effects of ultraviolet (UV) light, lack of skincare, male hormones, and secretion of anti-EGFR antibodies into the sweat.

UV rays are classified as UV-A, UV-B, and UV-C, based on their wavelengths. When the skin is exposed to UV-B radiation, photochemical reactions form cyclobutane-type pyrimidine dimers and (6-4)-adducts, which induce DNA damage and inflammatory reactions, thus decreasing epidermal barrier function $[15,16]$. Reduced epidermal barrier function leads to increased sensitivity to UV-B, making erythema more likely to develop even at low UV-B doses [17]. Moreover, the average UV-B levels and UV index in Japan (Tsukuba, 2014-2019) were reported to be $23.9 \mathrm{~kJ} / \mathrm{m}^{2}$ and 6.7, respectively, in summer and $6.3 \mathrm{~kJ} / \mathrm{m}^{2}$ and 2.0, respectively, in winter (Fig. 1b) [18]. Therefore, summer had higher UV-B levels and UV index than did winter. The UV index is formulated using the International Commission on Illumination reference action spectrum for UV-induced erythema on the human skin. For the average person, a UV index of 0 to 2 means low danger from the sun's UV rays. Moreover, a UV index of 6 to 7 means a high risk of harm from unprotected sun exposure [19]. The use of sunscreen can prevent erythema, DNA photodamage, and contact hypersensitivity [20]. Additionally, the application of sunscreen and general skincare products is significantly lower in men than in women [21,22].

Epithelial cells of sebaceous glands express androgen receptors, and sebaceous glands are target tissues for androgens [23]. Hence, androgenic species have been reported to interfere with the accumulation of structural proteins that rebuild damaged skin [24]. Furthermore, testosterone levels in men vary with 
season, with testosterone levels being lower in colder months than in hotter months [25]. Moreover, plasma testosterone levels in men are higher than those in women [26].

Based on the abovementioned findings, we hypothesized that factors such as increased UV radiation, lack of skincare, and high testosterone levels in summer could be responsible for the higher incidence of rashes in men than in women in summer. If these hypotheses are valid, skincare during the summer months is essential. Thus, focused patient education on the importance of skincare, particularly in the summer, may reduce the rash severity in patients, especially men, with CRC or HNC being treated with anti-EGFR antibodies.

Our retrospective study had several limitations. First, we could not investigate adherence to topical moisturizers and oral antibiotics, such as minocycline. However, we believe that patient adherence was acceptable, as we repeatedly explained the need for moisturizers and oral antibiotics at the start and continuation of treatment with anti-EGFR monoclonal antibodies. Second, there was a lack of information on the skin moisture content, sunscreen use, and testosterone levels of the included patients. Though our retrospective study examined as many factors as possible given the available resources, additional studies that consider the factors of adherence, skin moisture content, sunscreen use, and testosterone levels are needed to expand upon the findings of our study.

The novelty of our study is that we identified no difference in the incidence of rashes caused by treatment with anti-EGFR antibodies between the summer and winter months. However, men during the summer may be more prone to developing rashes than women during the summer as well as men and women during the winter.

The topic of our study is relevant to the current situation, as an increasing number of patients are receiving anti-EGFR therapy, making them susceptible to rashes. Rashes tend to be more frequent in men during summer. This information should help healthcare staff and patients take the necessary actions, such as education and practice of moisturizing, sunscreen use, and skin cleansing. However, rash mechanisms and preventive methods still remain unclear. We anticipate that the findings of our study on the relationship between rash and seasonal climate change will assist in the elucidation of rash mechanisms and establishment of preventive measures.

\section{Declarations}

\section{Acknowledgments}

This work was supported by Grant-in-Aid funding for general research from Gunma Prefectural Hospital (Grant number: C7-2019). We thank Mr. Masato Otsuka, General Affairs Division Manager, Gunma Prefectural Cancer Center for his assistance in preparing this manuscript. We would also like to thank EIGO EXPERTS for the English language editing.

\section{Authors' contributions}


Conceptualization, T.A. and Y.F.; methodology, T.A. and Y.F.; software, T.A. and Y.F.; validation, T.A. and Y.F.; formal analysis, T.A. and Y.F.; investigation, T.A., H.M., M.Y., E.H., and Y.A.; resources, T.A., H.M., M.Y., E.H., and Y.A.; data curation, T.A. and Y.F.; writing-original draft preparation, T.A., Y.F., H.I., H.M., M.Y., E.H., Y.A., H.O., H.H., K.M., and T.S.; writing-review and editing, T.A., Y.F., H.I., H.M., M.Y., E.H., Y.A., H.O., H.H., K.M., and T.S.; visualization, T.A. and Y.F.; supervision, Y.F., and H.I.; project administration, T.S.; funding acquisition, K.M. All authors have read and approved the published version of the manuscript.

\section{Additional information}

Competing interests: The authors declare no competing interests.

Data availability: The datasets generated during and/or analyzed during the current study are available from the corresponding author on reasonable request.

\section{References}

1. 1. Lordick, F. et al. Capecitabine and cisplatin with or without cetuximab for patients with previously untreated advanced gastric cancer (EXPAND): a randomised, open-label phase 3 trial. Lancet Oncol. 14, 490-499; 10.1016/s1470-2045(13)70102-5 (2013).

2. 2. Lynch T. J. et al. Epidermal growth factor receptor inhibitor-associated cutaneous toxicities: an evolving paradigm in clinical management. Oncologist 12, 610-621; 10.1634/theoncologist.12-5610 (2007).

3. 3. Lacouture, M. E. et al. Skin toxicity evaluation protocol with panitumumab (STEPP), a phase II, open-label, randomized trial evaluating the impact of a pre-emptive skin treatment regimen on skin toxicities and quality of life in patients with metastatic colorectal cancer. J. Clin. Oncol. 28, 1351$1357 ; 10.1200 / J C 0.2008 .21 .7828$ (2010).

4. 4. Kobayashi, Y. et al. Randomized controlled trial on the skin toxicity of panitumumab in Japanese patients with metastatic colorectal cancer: HGCSG1001 study; J-STEPP. Future Oncol. 11, 617-627; 10.2217/fon.14.251 (2015).

5. 5. Van Cutsem, E. et al. Open-label phase III trial of panitumumab plus best supportive care compared with best supportive care alone in patients with chemotherapy-refractory metastatic colorectal cancer. J. Clin. Oncol. 25, 1658-1664; 10.1200/JC0.2006.08.1620 (2007).

6. 6. Van Cutsem, E. et al. Intrapatient cetuximab dose escalation in metastatic colorectal cancer according to the grade of early skin reactions: the randomized EVEREST study. J. Clin. Oncol. 30, 2861-2868; 10.1200/JC0.2011.40.9243 (2012).

7. 7. Terms about time (Japan Meteorological Agency) (https://www.jma.go.jp/jma/kishou/know/yougo_hp/toki.html) (Accessed on December 17, 2020)

8. 8. Historical weather data (Japan Meteorological Agency) (http://www.data.jma.go.jp/gmd/risk/obsdl/index.php) (Accessed on December 17, 2020). 
9. 9. Kikuchi, K., Kobayashi, H., Le Fur, I., Tschachler, E. \& Tagami, H. The winter season affects more severely the facial skin than the forearm skin: comparative biophysical studies conducted in the same Japanese females in later summer and winter. Exog. Dermatol. 1, 32-38; 10.1159/000047989 (2002).

10. 10. Uenishi, T., Sugiura, H. \& Uehara, M. Changes in the seasonal dependence of atopic dermatitis in Japan. J. Dermatol. 28, 244-247; 10.1111/j.1346-8138.2001.tb00125.x (2001).

11. 11. Engebretsen, K. A., Johansen, J. D., Kezic, S., Linneberg, A. \& Thyssen, J. P. The effect of environmental humidity and temperature on skin barrier function and dermatitis. J. Eur. Acad. Dermatol. Venereol. 30, 223-249; 10.1111/jdv.13301 (2016).

12. 12. Lankheet, N. A. et al. The effect of seasonal variation and secretion of sunitinib in sweat on the development of hand-foot syndrome. Eur. J. Clin. Pharmacol. 69, 2065-2072; 10.1007/s00228-0131579-4 (2013).

13. 13. Gaiser MR, Lorenzen S, Merx K, Trojan J. \& Ocvirk J. Evaluation of EGFR inhibitor-mediated acneiform skin toxicity within the double-blind randomized EVITA trial: A thorough ex-specific analysis using the WoMo score. Cancer Med. 8, 4169-4175 (2019).

14. 14. Fisher DA. Hormone epidermal growth factor interactions in development. Horm Res. 33, 69-74 (1990).

15. 15. Mullenders, L. H. et al. UV-induced photolesions, their repair and mutations. Mutat. Res. 299, 271-276; 10.1016/0165-1218(93)90103-k (1993).

16. 16. Haratake, A. et al. UVB-induced alterations in permeability barrier function: role for epidermal hyperproliferation and thymocyte-mediated response. J. Invest. Dermatol. 108, 769-775; 10.1111/1523-1747.ep12292163 (1997).

17. 17. Takagi, Y., Mori K., Taguchi, H., Nishizaka, T. \& Takema, Y. UVB sensitivity correlates with cutaneous barrier function in the skin of Japanese females. Photodermatol. Photoimmunol. Photomed. 35, 284-285; 10.1111/phpp.12467 (2019).

18. 18. UV data collection (Japan Meteorological Agency) (http://www.data.jma.go.jp/gmd/env/uvhp/info_uv.html) (Accessed on December 17, 2020).

19. 19. World Health Organization. Global Solar UV Index: A Practical Guide. A joint recommendation of the World Health Organization, World Meteorological Organization, United Nations Environment Programme, and the International Commission on Non-lonizing Radiation Protection. https://www.who.int/uv/publications/en/UVIGuide.pdf (2002).

20. 20. Narbutt, J. et al. Sunscreen applied at $\geq 2 \mathrm{mg} \mathrm{cm}^{-2}$ during a sunny holiday prevents erythema, a biomarker of ultraviolet radiation-induced DNA damage and suppression of acquired immunity. Br. J. Dermatol. 180, 604-614; 10.1111/bjd.17277 (2019).

21. 21. Görig, T., Diehl, K., Greinert, R., Breitbart, E. W. \& Schneider, S. Prevalence of sun-protective behaviour and intentional sun tanning in German adolescents and adults: results of a nationwide telephone survey. J. Eur. Acad. Dermatol. Venereol. 32, 225-235; 10.1111/jdv.14376 (2018). 
22. 22. Gaiser, M. R. et al. Evaluation of EGFR inhibitor-mediated acneiform skin toxicity within the double-blind randomized EVITA trial: a thorough gender-specific analysis using the WoMo score. Cancer Med. 8, 4169-4175; 10.1002/cam4.2132 (2019).

23. 23. Lai, J. J., Chang, P., Lai, K. P., Chen, L. \& Chang, C. The role of androgen and androgen receptor in skin-related disorders. Arch. Dermatol. Res. 304, 499-510; 10.1007/s00403-012-1265-x (2012).

24. 24. Gilliver, S. C. \& Ashcroft, G. S. Sex steroids and cutaneous wound healing: the contrasting influences of estrogens and androgens. Climacteric 10, 276-288; 10.1080/13697130701456630 (2007).

25. 25. Demir, A., Uslu, M. \& Arslan, O. E. The effect of seasonal variation on sexual behaviors in males and its correlation with hormone levels: a prospective clinical trial. Cent. European J. Urol. 69, 285289; 10.5173/ceju.2016.793 (2016).

26. 26. Southren, A. L., Tochimoto. S., Carmody, N. C. \& Isurugi, K. Plasma production rates of testosterone in normal adult men and women and in patients with the syndrome of feminizing testes. J. Clin. Endocrinol. Metab. 25, 1441-1450; 10.1210/jcem-25-11-14 (1965).

\section{Tables}

Table 1. Patient characteristics. S group, patients who received treatment in the summer (June to August); W group, patients who received treatment in the winter (December to February); RDI, relative dose intensity; anti-EGFR mAb: anti-EGFR monoclonal antibody (cetuximab or panitumumab). 


\begin{tabular}{|c|c|c|c|c|c|}
\hline & \multicolumn{2}{|c|}{$S$ group $(N=34)$} & \multicolumn{2}{|c|}{$W$ group $(N=37)$} & \multirow[t]{2}{*}{$p$-value } \\
\hline & No. & $\%$ & No. & $\%$ & \\
\hline Sex & & & & & 0.32 \\
\hline Male & 20 & 58.8 & 26 & 70.3 & \\
\hline Female & 14 & 41.2 & 11 & 29.7 & \\
\hline \multicolumn{6}{|l|}{ Type of cancer } \\
\hline Colorectal cancer & 27 & 79.4 & 31 & 83.8 & 0.87 \\
\hline Head and neck cancer & 7 & 20.6 & 6 & 16.2 & \\
\hline \multicolumn{6}{|l|}{ Therapy regimen } \\
\hline Cetuximab-based regimen & 20 & 58.8 & 23 & 62.2 & 0.77 \\
\hline Panitumumab-based regimen & 14 & 41.2 & 14 & 37.8 & \\
\hline \multicolumn{6}{|l|}{ Moisturizer prescription } \\
\hline Yes & 34 & 100.0 & 37 & 100.0 & - \\
\hline No & 0 & 0.0 & & 0.0 & \\
\hline \multicolumn{6}{|l|}{ Minocycline prescription } \\
\hline Yes & 33 & 97.1 & 36 & 97.3 & 0.51 \\
\hline \multirow[t]{2}{*}{ No } & 1 & 2.9 & 1 & 2.7 & \\
\hline & \multicolumn{2}{|c|}{ Median (range) } & \multicolumn{3}{|c|}{ Median (range) } \\
\hline Age (years) & \multicolumn{2}{|c|}{$65(35-78)$} & \multicolumn{2}{|c|}{$64(35-78)$} & 0.45 \\
\hline RDI of anti-EGFR mAb & \multicolumn{2}{|c|}{$0.73(0.30-1.00)$} & \multicolumn{2}{|c|}{$0.74(0.39-1.07)$} & 0.80 \\
\hline
\end{tabular}

Figures 
a)

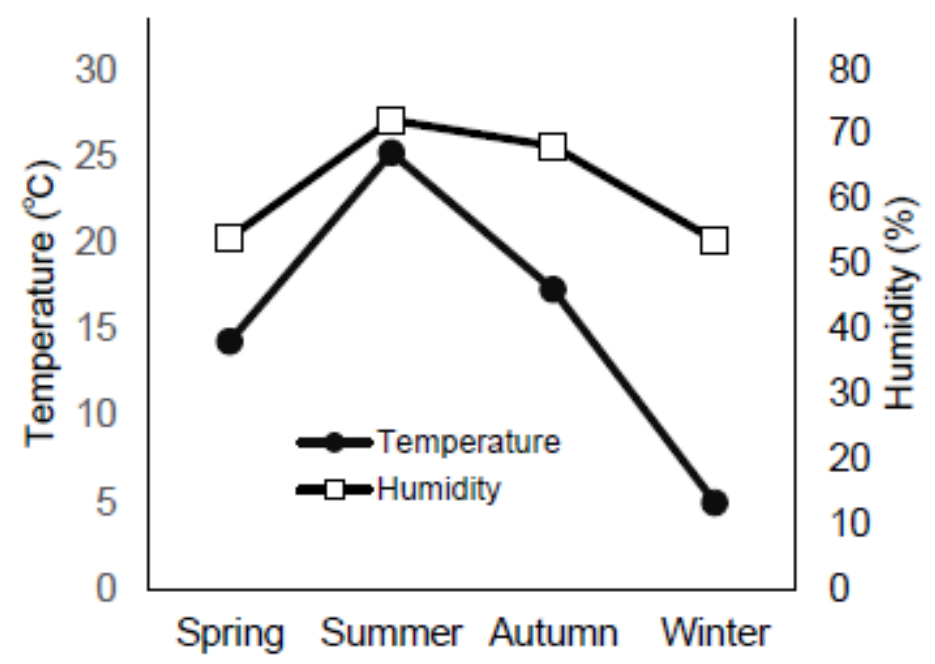

b)

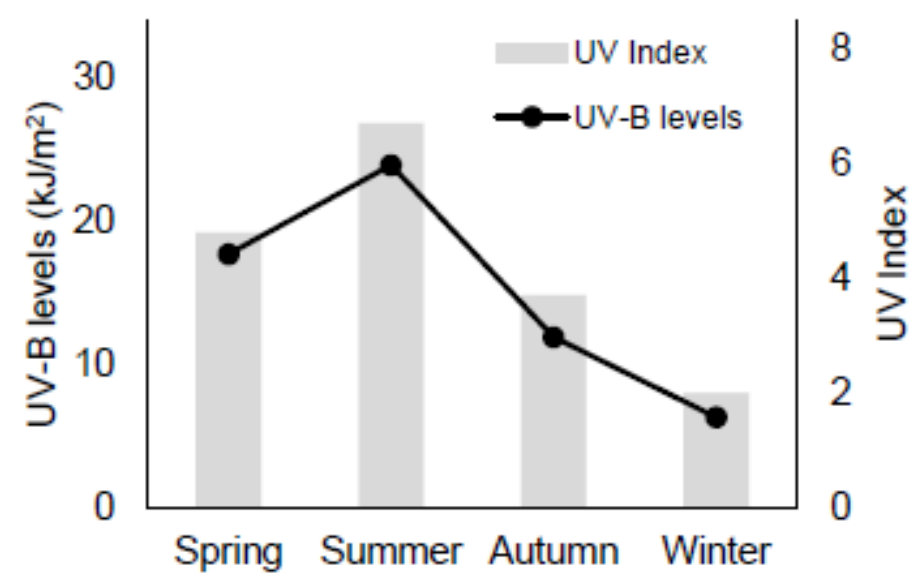

Figure 1

Observational data in different Japanese seasons. (a) The average ambient temperature $\left({ }^{\circ} \mathrm{C}\right.$; left axis) and average humidity (\%; right axis) (Maebashi, 2014-2019). Modified from "Historical weather data" with permission from the Japan Meteorological Agency. (b) The average ultraviolet (UV)-B levels (kJ/m2; at left axis) and average UV index (right axis) (Tsukuba, 2014-2019). Modified from “UV data collection" with permission from the Japan Meteorological Agency. 


\section{A total of 71 patients were assessed for eligibility}

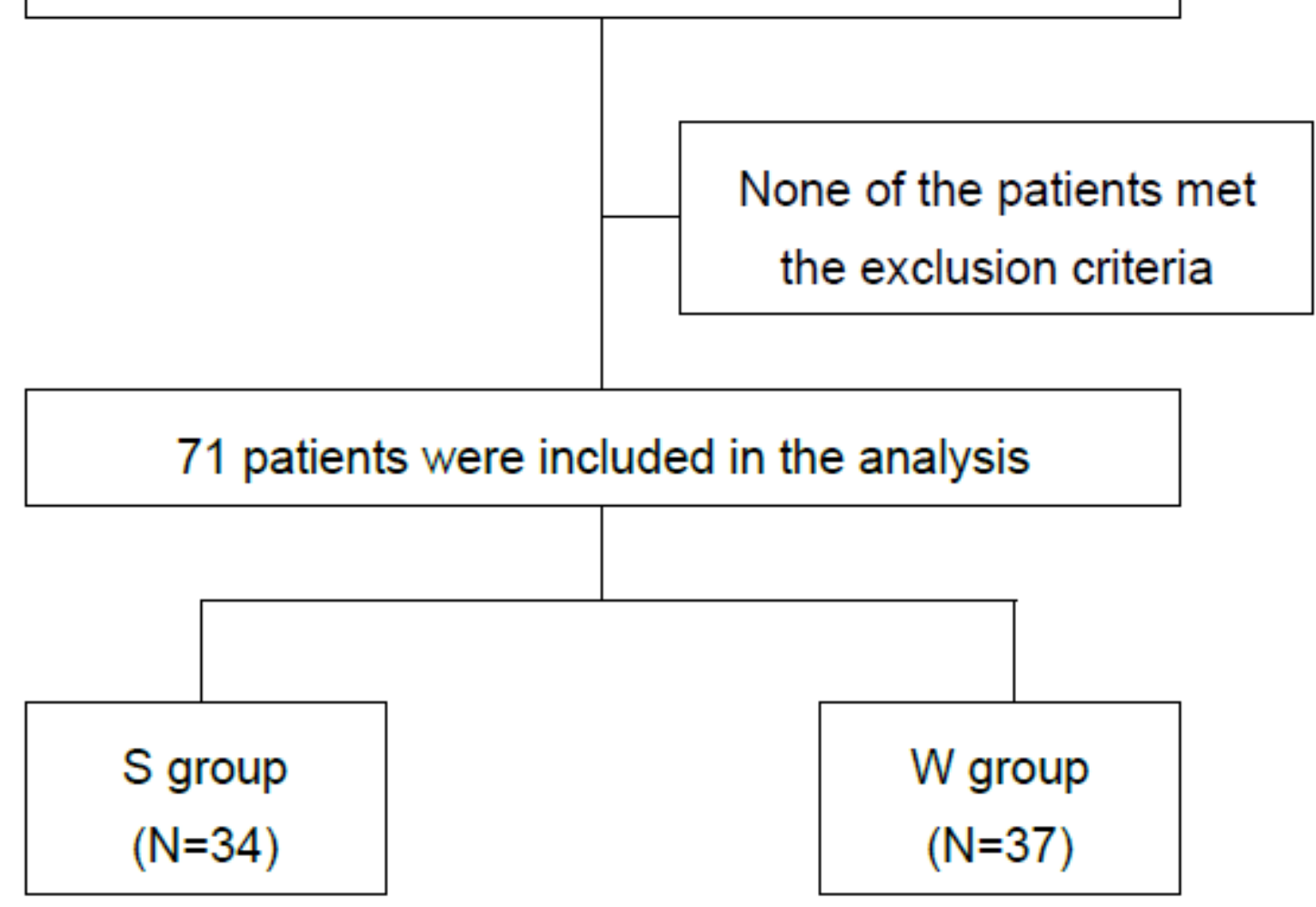

Figure 2

Consolidated Standards of Reporting Trials diagram for the study. S group, patients who received treatment in the summer (June to August); W group, patients who received treatment in the winter (December to February). 
a)

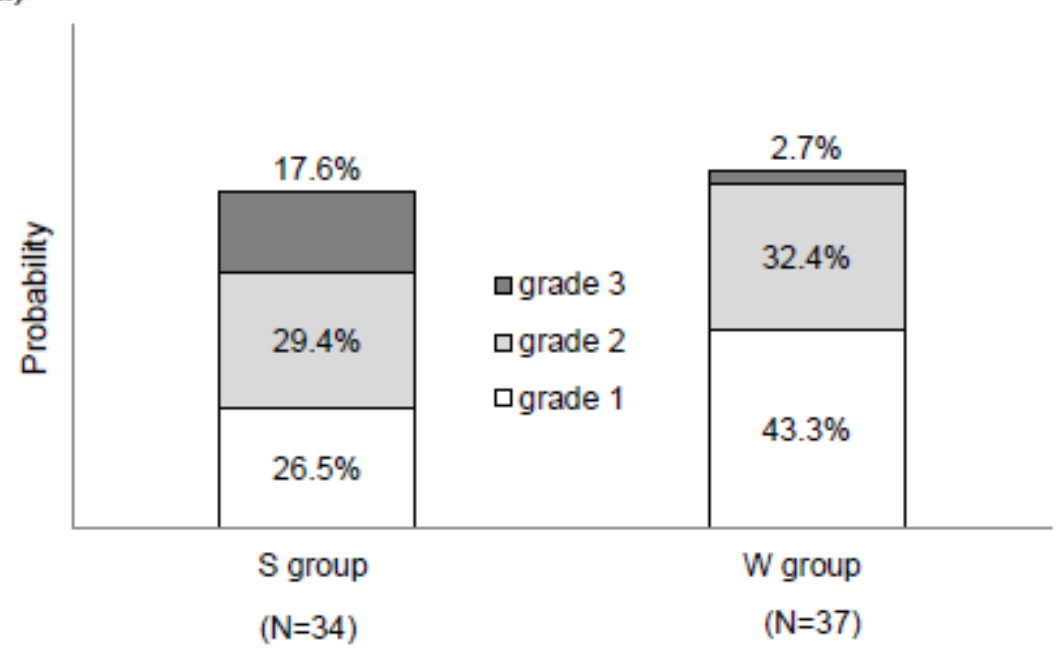

b)

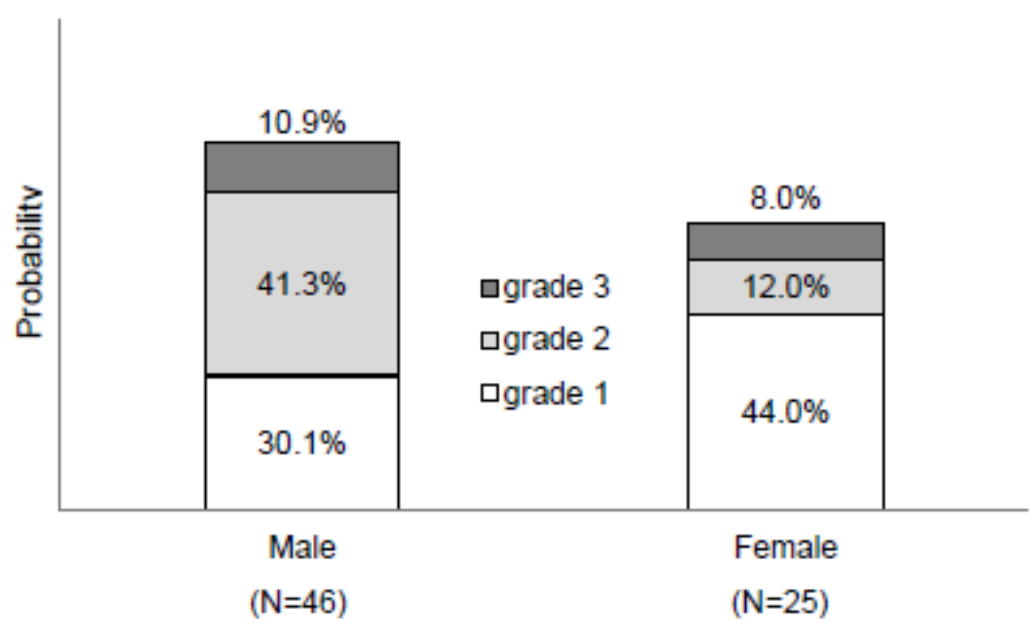

Figure 3

Incidence of acne-like rashes in patients with cancer treated with either cetuximab or panitumumab. (a) Incidence of acne-like rashes during the summer (S) and winter (W). (b) Incidence of acne-like rashes in men and women. 


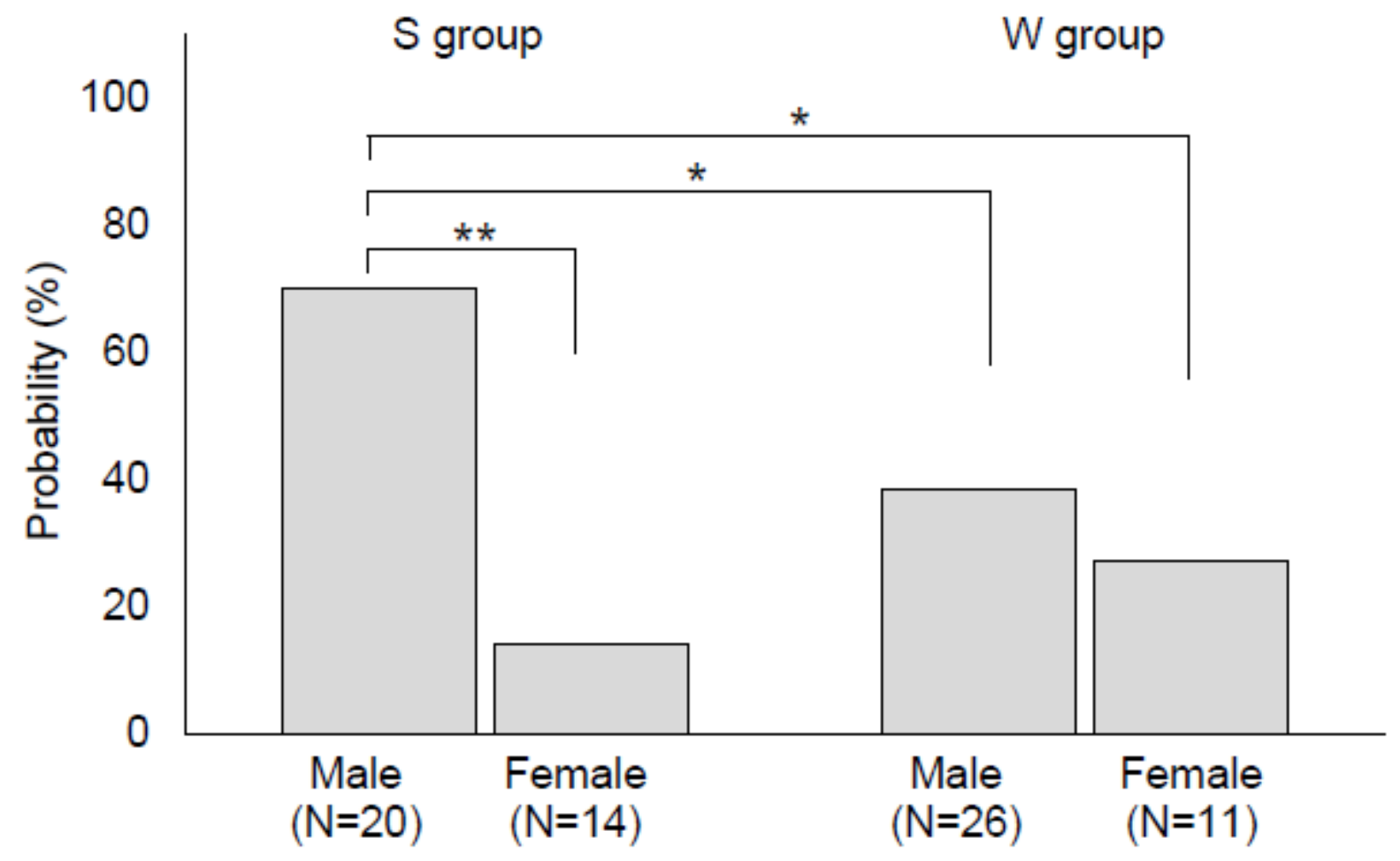

Figure 4

Incidence of grade 2 or higher acne-like rashes based on sex and season. Left, summer (S); right, winter (W). ${ }^{*} p<0.05,{ }^{* *} p<0.01$. 